Original

\title{
Patterned Electrical Neuromuscular Stimulation Decreases the Incidence of Incontinence in Elderly Patients
}

\author{
John Chris CASTEL
}

\begin{abstract}
The purpose of this study was to determine if Patterned Electrical Neuromuscular Stimulation (PENS) and Medium Frequency Alternating Current (MFAC) would decrease the incidence of urinary incontinence (UI) in institutionalized elderly patients. The study subjects comprised 55 women (mean age of 85.14 years) who were residents of skilled nursing facilities. All patients received three, 20-minute PENS treatments per week for 6 weeks, applied to the T10-L2 paraspinal and suprapubic abdominal muscles as well as to the L1-2 and Sacral S2-S4 proximal medial thigh dermatomes and muscles. Additionally, 16 of the patients also received MFAC to the pelvic floor for 10 minutes immediately after the PENS treatment. We compared incidences of UI prior to and after the program in subjects who suffered from urge or mixed types of UI. On average, subjects in the PENS only group improved $73 \%$, whereas subjects in the PENS and MFAC group improved $66 \%$. Overall, $92 \%$ of the patients reported satisfaction with the incontinence program and would recommend this treatment to others who are incontinent. The follow-up progress notes of 18 patients were reviewed 4 weeks post discharge and only one patient did not maintain the improved status. Both PENS and MFAC are effective in treating patients with UI. PENS achieves the neuromuscular re-education of the pelvic muscle complex and modulates the urge sensation; whereas MFAC is used to strengthen the pelvic floor and sphincter muscles.
\end{abstract}

Key words : urinary incontinence, neuromuscular electrical stimulation

\section{Introduction}

Urinary incontinence (UI) is a pervasive problem in long-term care populations. It is estimated that $18 \%$ of non-institutionalized and $50 \%$ of institutionalized patients above the age of 65 suffer some form of $\mathrm{UI}^{1)}$. At least one-half of the 1.5 million nursing home residents in the United States are plagued by UI of varying etiology. Stress UI, the involuntary loss of urine occurring when intravesical pressure exceeds the maximum urethral pressure, is often caused by pelvic floor and sphincter muscle weakness and dysfunction of the proprioceptive motor control loops between the sphincter and the pelvic floor. This can occur with rapid increases in intra-abdominal pressure associated with physical activity, laughing and coughing ${ }^{2)}$. Urge incontinence, the most common form of UI among the

Department of Rehabilitation Medicine Showa University School of Medicine, 1-5-8 Hatanodai Shinagawa-ku, Tokyo 142-8666, Japan. 
elderly, is an inability to delay voiding after the sensation of bladder fullness is perceived ; it is frequently associated with detrusor hyperactivity, and is most often encountered following stroke, dementia and neural sensitization. Detrusor hyperactivity in the elderly exists as two physiologically distinct subsets, one in which detrusor contractile function is preserved and one in which it is impaired. Detrusor instability with impaired contractility mimics virtually all other types of established incontinence. Urinary retention secondary to cholinergic agents occurs more frequently in patients with impaired contractility than in those with normal detrusor function, limiting the efficacy of those drugs ${ }^{3)}$.

Like many geriatric conditions, UI is frequently a multifactorial problem. A substantial proportion (as many as one-third) of older incontinent women with symptoms and urodynamic findings consistent with stress UI also have some irritative voiding symptoms and detrusor hyperactivity on urodynamic testing. A large proportion of the frail elderly population with UI has both urge incontinence with detrusor hyperactivity on urodynamic testing and stress incontinence symptoms. They may also have additional neurologic impairments that contribute to their urinary incontinence. This group of the population is referred to as mixed incontinence and is by far the largest group of older incontinent women.

Overflow UI, less common among the elderly, may result from anatomic or neurogenic outflow obstruction. Neurological conditions, such as spinal cord injury, multiple sclerosis and diabetic neuropathy may result in an under-active, high compliance bladder and abnormal sphincter activity known as neurogenic UI. Finally, if the UI is related to factors outside the lower urinary tract, such as a physical inability to get to a bathroom in a timely manner, it is considered functional.

The financial cost of incontinence is significant and is estimated to be in excess of $\$ 27.9$ billion in the United States alone ${ }^{1}$. This can be broken down into $\$ 3$ billion for routine costs in institutional settings, $\$ 7$ billion for routine costs for patients living in the community, $\$ 1.5$ billion in adult incontinence products and laundry costs, and $\$ 12.4$ billion for adverse consequences of incontinence including skin breakdown, infection, falls, longer hospital stays and increased admission to nursing homes. From the provider's perspective, it is estimated that, in 1987, the industry spent an average of $\$ 4,104(\$ 7,797$ in 1998) per incontinent elderly nursing home resident, a figure that is more than $10 \%$ of annual total nursing home expenditure. Direct operational costs of managing UI (e.g., supplies, laundry and labor) range from $\$ 3.77$ ( $\$ 7.16$ in 1998) to $\$ 11.09$ ( \$21.07 in 1998) per patient per day. In addition, the patients often suffer numerous psychological consequences such as fear of being odorous, sexual dysfunction, and embarrassment associated with the incontinence.

Current methods of treatment of the frail elderly include surgical intervention, pharmacological and fluid management, pelvic floor exercises and behavioral modification such as biofeedback, pelvic muscle neuro-muscular stimulation-prompted voiding and assisted voiding ${ }^{4)}$. These methods produce inconsistent results in elderly institutionalized patients due to the medically complex nature of these patients, the lack of definitive diagnosis using urodynamic testing and the degree of neurologic impairment generally present. As a result, UI largely goes untreated as systematized methods of care have not been developed or applied to long-term care institutionalized patients. The institutionalized setting also poses significant problems relative to outcome assessment, as monitoring of episodes of 
incontinence is practically difficult in these settings.

Improvements to electro-stimulation methods of treatment for UI have been developed over the last three years for incontinence management programs focused on the elderly. A new device (Omnistim FX ${ }^{2}$, Accelerated Care Plus Corp., Reno NV, USA) uses Patterned Electrical Neuromuscular Stimulation (PENS) to treat incontinence; it is based on the re-education of pelvic motor-sensory systems. Recent improvements in another method of treatment, Medium Frequency Alternating Current therapy (MFAC), have also shown promising results. MFAC targets the treatment of disuse muscle atrophy in the pelvic muscle complex. The PENS and MFAC techniques are easy to apply, and patient compliance is high since the application is minimally invasive and comfortable. These methods are also highly suitable for "tuning up the proprioceptive and motor systems" prior to and during the application of voluntary pelvic muscle exercise and biofeedback.

This study examined the effect of PENS and MFAC treatment on institutionalized cognitive elderly patients suffering from incontinence. We limited our study to the two most common types of urinary incontinence presenting in the frail elderly, mixed and urge, however we also discuss several case studies that illustrate the potential mechanisms of PENS therapy, which were closely monitored using urodynamic testing methods.

\section{Methods}

\section{Subjects}

Fifty-five female subjects with a mean age of 85.14 years participated in the study. We explained the methods and obtained written consent from each subject prior to data collection. All subjects lived in skilled nursing facility residences (SNF). Prior to participation in the program, a patient history form was completed by the facility's rehabilitation and nursing staff. To ensure that the underlying reason for incontinence episodes was not due to a lack of mobility (Functional Incontinence), patients were assessed on transfer items (transfers: bed, chair, and wheelchair) on the Functional Independence Measure (FIM). All patients participating in this program were able to perform $50 \%$ to $74 \%$ of transferring tasks with only moderate assistance, representing an average of three FIM points.

\section{Instruments}

Mini Mental Status Exam (MMSE). The MMSE is a 30-point scale designed to assess a patient's cognitive performance (orientation, attention, memory, and language) in a clinical setting. Incontinence questionnaires, diaries and patient satisfaction surveys were developed to allow systematic evaluation of subjective and objective outcome measures. Urodynamic testing was performed with several instruments; the Lumax fiber-optic cystometry and uroflow measurement system and the Diagnostic Ultrasound Udx device, which was used to measure post-void residual urine. Urinary tract infection was assessed using Chemstrips - urinalysis and follow-up laboratory testing if indicated. Pelvic muscle performance was evaluated by dual channel Electromyographic (EMG) recording, which assesses both frequency spectral analysis and amplitude.

The device used to provide PENS and pelvic floor neuromuscular stimulation was the Omnistim $\mathrm{FX}^{2}$ (Accelerated Care Plus, Reno, NV). This device has two modes, 1) Patterned Electrical Nerve Stimulation (PENS) for neuromuscular re-education of the pelvic 


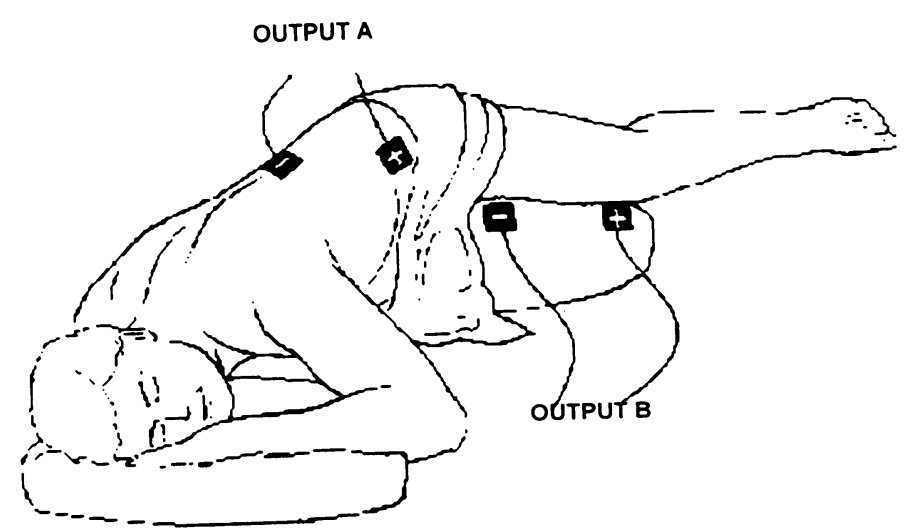

Fig. 1. Electrode placement for PENS application for treatment of urinary incontinence

muscle complex and to modulate urge sensation; and 2) medium frequency alternating current (MFAC) for stimulation of the pelvic floor and sphincter muscles, which is used to decrease muscle disuse atrophy.

\section{Clinical interventions}

Each patient was first administered the MMSE as part of the inclusion criteria. A score of 20 or greater was considered appropriate for inclusion in the study as the patients were considered sufficiently cognitive to participate in the questionnaires and data collection. MMSE was not considered as an exclusion criteria for reasons of clinical efficacy, simply for the ability to report data during the study period. A urinary incontinence questionnaire was completed and data was collected using voiding diaries and a patient satisfaction survey at the end of the study period. The patients were classified as having either mixed, or urge types of incontinence according to their results on the questionnaire, their patient history and symptoms. A small group of patients reported in the case studies were evaluated with Urodynamic testing and measurement of post-void residual urine. All patients were tested for UTI using Chem strips and placed on antibiotics prior to the start of care if required.

We collected data in three phases. Phase 1 included 39 subjects with PENS as the only treatment intervention. Each patient received three 20-minute treatments per week for 6 weeks. The $\mathrm{FX}^{2}$ PENS was applied to the T10-L2 para spinal and suprapubic abdominal muscles, and to the L1-2 and sacral S2-S4 proximal medial thigh dermatomes and muscles (Fig. 1). The stimulus parameters were preset at 60 uSec pulses separated into $200 \mathrm{mSec}$ pulse trains at $50 \mathrm{HZ}$. There was a 1.2 second pause between stimulus cycles and a 160 $\mathrm{mSec}$ delay from the beginning of the first channel applied to the flank and abdomen and the second applied to the thigh. The stimulation pattern mimics the normal physiologic tri-phasic EMG pattern associated with lower extremity motor firing of agonist and antagonist muscles. The intensity was increased to obtain a minimal muscle twitch of the thoraco-lumbar para spinal muscles and lower abdominal muscles, and the medial proximal thigh muscles. During the first two weeks, the current was set at a sensory level (no visible twitch but a patient report of sensation). Thereafter and for the rest of the treatments, stimulation was set to a visible muscle twitch level. Average current per pulse was $55 \mathrm{~mA}$ 


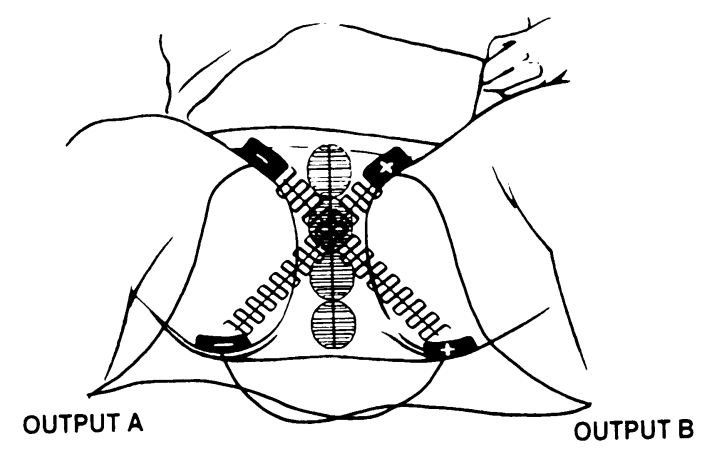

Fig. 2. Electrode placement for pelvic floor muscle strengthening with MFAC

and was well tolerated by all patients but one.

Phase two of the experiment included 16 patients who received both PENS and MFAC. The PENS treatments were administered the same way as for phase one during the entire six weeks. Starting the third week and continuing through to the sixth week, MFAC treatments were administered immediately after the PENS treatments. For the MFAC treatment, four electrodes were applied over the gluteal and lower abdominal muscles, and the current was passed through in a crossing pattern creating a vector field over the pelvic floor and sphincters (Fig. 2) ${ }^{5}$. The intensity was turned up to motor threshold level and the amplitude was adjusted to contract the pelvic muscles during the "on" phase of the current. Stimulation was applied at $2500 \mathrm{~Hz}$ frequency modulated at 50 bursts per second (BPS) with an "on" time of 10 seconds and an "off" time of 50 seconds with on and off ramps of 2 seconds. The treatment time was 10 minutes, which provided ten contractions. Patients were requested to contract voluntarily in conjunction with the electrically induced contractions if they were capable of doing so. This protocol has been well established in the literature to strengthen enervated muscle ${ }^{6}$.

Phase III of the study involved a group of fifteen cases, two of which are reported in this article to illustrate the potential mechanisms of action of PENS. Outcome tracking using the methods of phase I and II, as well as Urodynamic testing on admission and monitoring of Uroflow and post-void residual at various times during the course of treatment, were used. In addition, more detailed reporting instruments were developed for assessing subjective and objective symptoms of incontinence including sensation, urge characterization and full history and physical evaluation of the patients. Phase III study participants were all treated with PENS and MFAC (if appropriate for treatment of muscle disuse atrophy). Electromyographic assessment was used on some of the patients to detect any changes in pelvic floor muscle disuse atrophy and abdominal-pelvic muscle dysinergy. Upon discharge, all patients completed a satisfaction survey. Four weeks post-discharge from the program; follow-up progress notes were reviewed to determine the efficacy of the treatment.

\section{Results}

The results of phases one and two are included in Tables 1 and 2, respectively. Of the 39 subjects who underwent the PENS-only treatment, six were classified as having "urge" UI 
Table 1.

Mixed incontinence PENS and MFAC

\begin{tabular}{|c|c|c|c|c|c|c|c|c|c|}
\hline Age & Sex & Type of UI & $\begin{array}{c}\text { Mini } \\
\text { Mental }\end{array}$ & $\begin{array}{c}\text { \# NMES } \\
\text { Rx. }\end{array}$ & $\begin{array}{c}\text { \# MFAC } \\
\text { Rx. }\end{array}$ & $\begin{array}{c}\text { \# UI Prior } \\
\text { Rx. }\end{array}$ & $\begin{array}{l}\text { \# UI After } \\
\text { Rx. }\end{array}$ & $\begin{array}{c}\text { Decrease/ } \\
\text { Day }\end{array}$ & $\%$ Decrease \\
\hline 65 & $\mathbf{F}$ & $\mathbf{M}$ & & 6 & 6 & 12 & 0 & 12 & $100 \%$ \\
\hline 89 & F & $\mathbf{M}$ & & 7 & 7 & 13 & 6 & 7 & $54 \%$ \\
\hline 94 & F & $\mathbf{M}$ & & 28 & 10 & 2.5 & 0.5 & 2 & $80 \%$ \\
\hline 77 & $\mathrm{~F}$ & $\mathbf{M}$ & & 30 & 10 & 8 & 6 & 2 & $25 \%$ \\
\hline 66 & F & $\mathbf{M}$ & 30 & 19 & 15 & 8 & 5 & 3 & $38 \%$ \\
\hline 92 & F & $\mathbf{M}$ & 27 & 20 & 17 & 7 & 3 & 4 & $57 \%$ \\
\hline 86 & F & $\mathbf{M}$ & & 20 & 20 & 10 & 0 & 10 & $100 \%$ \\
\hline 74 & F & $\mathbf{M}$ & 30 & 22 & 22 & 8 & 2 & 6 & $75 \%$ \\
\hline 90 & F & $\mathbf{M}$ & & 40 & 24 & 9 & 0 & 9 & $100 \%$ \\
\hline 85 & F & $\mathbf{M}$ & 20 & 25 & 25 & 7 & 2 & 5 & $71 \%$ \\
\hline 79 & F & $\mathbf{M}$ & 28 & 26 & 26 & 7 & 2 & 5 & $71 \%$ \\
\hline 67 & F & $\mathbf{M}$ & 20 & 28 & 28 & 4 & 1 & 3 & $75 \%$ \\
\hline 80 & $\mathrm{~F}$ & $\mathbf{M}$ & 30 & 35 & 35 & 7 & 2 & 5 & $71 \%$ \\
\hline 92 & F & $\mathbf{M}$ & & 35 & 35 & 6 & 2 & 4 & $67 \%$ \\
\hline 75.73 & $\mathbf{F}$ & $\mathbf{M}$ & 26.43 & 22.73 & 18.67 & 7.23 & 2.10 & 5.13 & $66 \%$ \\
\hline \multicolumn{10}{|c|}{ PENS only } \\
\hline 83 & $\mathrm{~F}$ & $\mathbf{M}$ & 28 & 22 & & 3 & 1 & 2 & $67 \%$ \\
\hline 81 & F & $\mathbf{M}$ & & 32 & & 6 & 2.5 & 3.5 & $58 \%$ \\
\hline 77 & F & $\mathbf{M}$ & & 34 & & 3 & 1 & 2 & $67 \%$ \\
\hline 90 & F & $\mathbf{M}$ & & 34 & & 4 & 3 & 1 & $25 \%$ \\
\hline 90 & F & $\mathbf{M}$ & 30 & 18 & & 2 & 0.5 & 1.5 & $75 \%$ \\
\hline 89 & F & $\mathbf{M}$ & & 12 & & 10 & 2 & 8 & $80 \%$ \\
\hline 87 & F & $\mathbf{M}$ & 30 & 15 & & 1 & 0 & 1 & $100 \%$ \\
\hline 85 & $\mathrm{~F}$ & $\mathbf{M}$ & 28 & 16 & & 2 & 0 & 2 & $100 \%$ \\
\hline 78 & F & $\mathbf{M}$ & & 40 & & 10 & 0 & 10 & $100 \%$ \\
\hline 91 & F & $\mathbf{M}$ & 19 & 25 & & 22 & 6 & 16 & $73 \%$ \\
\hline 85 & $\mathrm{~F}$ & $\mathbf{M}$ & & 25 & & 5 & 1 & 4 & $80 \%$ \\
\hline 85 & $\mathbf{F}$ & $\mathbf{M}$ & & 30 & & 6 & 0 & 6 & $100 \%$ \\
\hline 77 & $F$ & $\mathbf{M}$ & & 25 & & 10 & 0 & 10 & $100 \%$ \\
\hline 91 & F & $\mathbf{M}$ & & 24 & & 5 & 3 & 2 & $40 \%$ \\
\hline 79 & F & $\mathbf{M}$ & & 25 & & 6 & 0 & 6 & $100 \%$ \\
\hline 70 & F & $\mathbf{M}$ & 29 & 25 & & 8 & 4 & 4 & $50 \%$ \\
\hline 84 & F & $\mathbf{M}$ & & 10 & & 13 & 10 & 3 & $23 \%$ \\
\hline 88 & $\mathbf{F}$ & $\mathbf{M}$ & 28 & 35 & & 11 & 0 & 11 & $100 \%$ \\
\hline 92 & $\mathbf{F}$ & $\mathbf{M}$ & & 30 & & 3 & 2 & 1 & $33 \%$ \\
\hline 85 & F & $\mathbf{M}$ & 29 & 36 & & 5 & 3 & 2 & $40 \%$ \\
\hline 75 & F & $\mathbf{M}$ & 25 & 30 & & 15 & 9 & 6 & $40 \%$ \\
\hline 86 & F & $\mathbf{M}$ & 23 & 35 & & 8 & 0.5 & 7.5 & $94 \%$ \\
\hline 70 & F & $\mathbf{M}$ & 20 & 18 & & 7 & 0 & 7 & $100 \%$ \\
\hline 90 & F & $\mathbf{M}$ & & 19 & & 15 & 11 & 4 & $27 \%$ \\
\hline 89 & $\mathbf{F}$ & $\mathbf{M}$ & & 24 & & 2 & 0 & 2 & $100 \%$ \\
\hline 84 & $\mathbf{F}$ & $\mathbf{M}$ & & 33 & & 3 & 1 & 2 & $67 \%$ \\
\hline 91 & F & $\mathbf{M}$ & & 27 & & 8 & 3 & 5 & $63 \%$ \\
\hline 92 & F & $\mathbf{M}$ & & 25 & & 5 & 0 & 5 & $100 \%$ \\
\hline 70 & F & $\mathbf{M}$ & 30 & 42 & & 15 & 0 & 15 & $100 \%$ \\
\hline 76 & F & $\mathbf{M}$ & & 37 & & 4 & 0 & 4 & $100 \%$ \\
\hline 86 & F & $\mathbf{M}$ & & 30 & & 12 & 1 & 11 & $92 \%$ \\
\hline 81 & F & $\mathbf{M}$ & & 40 & & 8 & 6 & 2 & $25 \%$ \\
\hline 72 & F & $\mathbf{M}$ & 23 & 20 & & 1 & 0.7 & 0.3 & $30 \%$ \\
\hline 85.91 & $\mathbf{F}$ & $\mathbf{M}$ & 26.15 & 27.91 & & 7.44 & 2.23 & 5.21 & $73 \%$ \\
\hline
\end{tabular}


Incontinence PENS

Table 2. URGE

MFAC

\begin{tabular}{|c|c|c|c|c|c|c|c|c|c|}
\hline Age & Sex & Type of UI & $\begin{array}{c}\text { Mini } \\
\text { Mental }\end{array}$ & $\begin{array}{c}\text { \# NMES } \\
\text { Rx. }\end{array}$ & $\begin{array}{c}\text { \# MFAC } \\
\text { Rx. }\end{array}$ & $\begin{array}{l}\text { \# UI Prior } \\
\text { Rx. }\end{array}$ & $\begin{array}{l}\text { \# UI After } \\
\text { Rx. }\end{array}$ & $\begin{array}{c}\text { Decrease/ } \\
\text { Day }\end{array}$ & $\%$ Decrease \\
\hline 85 & $\mathrm{~F}$ & $\mathrm{U}$ & & 15 & 9 & 6 & 0 & 6 & $100 \%$ \\
\hline 83 & $\mathrm{~F}$ & $\mathrm{U}$ & 30 & 48 & 38 & 22 & 1.5 & 0.7 & $32 \%$ \\
\hline 84 & $\mathbf{F}$ & $\mathbf{U}$ & & 31.5 & 23.5 & 4.1 & 0.75 & 3.35 & $66 \%$ \\
\hline \multicolumn{10}{|l|}{$\begin{array}{l}\text { PENS } \\
\text { only }\end{array}$} \\
\hline 74 & $\mathrm{~F}$ & $\mathrm{U}$ & & 25 & & 9 & 5 & 4 & $44 \%$ \\
\hline 80 & $\mathrm{~F}$ & $\mathrm{U}$ & 25 & 17 & & 5 & 1 & 4 & $80 \%$ \\
\hline 83 & $\mathrm{~F}$ & $\mathbf{U}$ & & 25 & & 20 & 11 & 9 & $45 \%$ \\
\hline 88 & $\mathbf{F}$ & $\mathbf{U}$ & & 30 & & 2 & 0.2 & 1.8 & $90 \%$ \\
\hline 73 & $\mathrm{~F}$ & $\mathrm{U}$ & & 30 & & 4.7 & 1.3 & 3.4 & $72 \%$ \\
\hline 79 & $\mathrm{~F}$ & $\mathrm{U}$ & & 34 & & 13 & 0.01 & 12.99 & $100 \%$ \\
\hline 79.50 & $\mathbf{F}$ & $\mathbf{U}$ & & 26.83 & & 8.95 & 3.09 & 5.87 & $72 \%$ \\
\hline
\end{tabular}

and the other 33 as having "mixed" UI. In the mixed incontinence group, those receiving PENS only had an average of 7.44 incidences of UI per day prior to the 6-week treatment. After the 6-week treatment, this decreased to 2.23 incidences of UI per day, which represents an improvement of $73 \%$. In the same group, those receiving PENS and MFAC had an average of 7.23 incidences of UI per day prior to the 6-week treatment, and 2.10 incidences per day after the 6-week treatment; this is an improvement of $66 \%$ (Table 1). In the urge incontinence group, those receiving PENS only had an average of 8.95 incidences of UI per day prior to the 6-week treatment, and 3.09 incidences per day after the 6-week treatment, representing an improvement of $72 \%$. Those receiving PENS and MFAC in the urge incontinence group had an average of 4.1 incidences of UI per day prior to the 6-week treatment, and 0.75 incidences per day after the 6-week treatment period, representing an improvement of $66 \%$ (Table 2 ).

We also collected data on various components of UI such as urge to urinate, or having to get up during the night to void the bladder. With both treatments, the urge/need to urinate decreased. On admission, 26 of the patients felt the urge/need to urinate every two hours on average and this increased to every three hours on average upon discharge from the program. For all types of incontinence, the average number of times the patients needed to get up in the middle of the night to urinate decreased with treatment. All 24 patients who answered this item reported an average of three times per night, whereas upon discharge the number had decreased to two times each night. The frequency of losing urine also decreased from two times a day to once per day.

On admission to the program, $31 \%$ reported having a problem with bowel continence and upon discharge $12 \%$ reported that bowel incontinence was still a problem. There was also a $19 \%$ decrease in subjects reported constipation as a problem as compared to the time of admission to the program.

The satisfaction survey revealed that $100 \%$ of the patients did not feel any pain during 
any of the treatment. Also, $84 \%$ of the patients reported that they did not have as many incontinence episodes, $40 \%$ reported that they participated in more activities, $86 \%$ reported easier daily care, and $68 \%$ reported that they felt they could take care of their own daily hygiene. Overall, $92 \%$ of the patients reported satisfaction with the incontinence program and $92 \%$ reported that they would recommend this treatment to others who are incontinent. The follow-up progress notes of 18 patients were reviewed 4 weeks post-discharge from the program and the results revealed that only one patient did not maintain the improved status.

\section{Case study results with urodynamic testing}

Case I Detrusor Sphincter Dysenergy

The patient was a 60-year-old female with Intrinsic Sphincter Dysfunction (ISD) and Detrusor Sphincter Dyssynergy (DSD) determined through urodynamic testing. She considered her incontinence to be a major problem (8-9 on a visual analog scale). Her MMSE was 29 and the patient was motivated to improve. The incontinence started three months prior to the start of care with sudden onset and with significant urge sensation. Her incontinence episodes occurred immediately following urge sensation on the way to the toilet, when sitting up and approximately three times each night. The patient suffered from insomnia. Protection (diaper or pad) was wet, not damp, indicating significant fluid loss on incontinent voiding. The patient had a starting post-void residual of $137 \mathrm{ml}$ on initiation of PENS treatment. The patient indicated difficulty in starting her urine stream. This was measured with uroflow instrumentation at the start and completion of therapy. Hesitancy was measured at 112 seconds, and void time was 14 seconds with an average flow rate of $7.1 \mathrm{ml} / \mathrm{sec}$. PENS was given 3 times per week for 5 weeks according to the standard protocol. Within two weeks of starting treatment, the patient described that she could initiate her stream faster and had made it through the weekend without any incidence of incontinence during the evenings. By the end of the five-week course of treatment, hesitancy was measured at 82 seconds (a reduction of 40 seconds or $36 \%$ ) and void time was measured at 13 seconds with an average flow rate of $10.6 \mathrm{ml} / \mathrm{sec}$ (an increase of 3.5 $\mathrm{ml} / \mathrm{sec}$ or $49 \%$ ). Post-void residual was measured at $60 \mathrm{ml}$ (a decrease of $77 \mathrm{ml}$ or $56 \%$ ). The patient no longer experienced accidents or nighttime incontinent voids. Urge sensation was not as intense and could be controlled by the patient sufficiently to void without incontinence on the way to the toilet. At the end of the course of treatment, assessment with EMG indicated that the patient was able to recruit sphincter and pelvic floor muscles independently of abdominal contraction (no co-contraction indicating reduced dysinergy). In addition, the fast twitch fibers were moderately recruited. The patient was referred to a sleep disorder program following her course of treatments for incontinence management.

\section{Case 2 Neurological Incontinence-Weak Bladder Secondary to MS}

The patient was a 60-year-old female with a 20-year history of MS. Upon urodynamic testing the patient demonstrated a high-capacity, high-compliance bladder (hypotonicity) with high post-void residual. The patient had no micturition sensation prior to or during urination. The MMSE was 30. The patient was being treated with Ditropan (an inhibitor of urge). Based on the urodynamic testing results Ditropan was immediately discontinued (as it often contributes to increased bladder hypotonicity, which further increases urine retention in patients with hypotonic bladders). The patient was catheterized three times 
daily and frequently suffered urinary tract infection. The patient was routinely wet but could not identify the time or number of incidences. The patient received a total course of 30 PENS treatments according to the standard protocol over a five-month period. Little change in sensation or incontinence was observed over the first five weeks of treatment. Post-void residuals were monitored weekly as the patient was being catheterized three times daily at the start of care. The PVRs oscillated significantly from the initiation of treatment through the first three weeks. At the end of the third week of treatment, the patient reported being able to sense fullness about once a day. Three months following the start of care, the patient had normal sensation of fullness and micturition, and was able to initiate voiding when placed on the toilet. Her plan of care was altered to catheterization once daily in the morning with prompted voiding three times daily without incontinence. Her post-void residual began decreasing during this period and continued downward as the patient was monitored over the next several months. It is interesting to note that as the patient began to regain micturition sensation the amplitude of the PENS stimulation required to reach motor threshold decreased significantly to 45 ma average output per channel, from the starting point of $74 \mathrm{ma}$ average per channel. This would indicate return of sensation due to decreased nerve stimulation threshold.

\section{Discussion}

This discussion section will address three areas. First, an overview of bladder function and physiology will be provided with a discussion of the potential mechanisms of patterned stimulation and MFAC. Secondly, the study data will be reviewed and, finally, the implications of our research for the treatment of urinary incontinence in the elderly and directions for future study will be discussed.

\section{Basic bladder function}

Urinary bladder control is coordinated by sensory stretch receptors in the bladder wall that provide volume or stretch information to the spinal circuits and brain. The afferent nerves enter the spine at T-10 to L-2 and S-2 to S-4 and increase in activity with increased stretch. This neurological input is variable in range with an upward trend as the stretch receptors are activated and become accommodated. At a certain threshold, the increased sensory input can no longer be inhibited by the higher centers and the voiding reflex occurs.

The bladder contracts by activation of the para-sympathetic nerves at S-2, 3, and 4, which are the nerves that innervate the bladder dome (detrusor muscle). The bladder neck relaxes (internal sphincters), as do the external sphincter muscles (pudendal enervation) ${ }^{7}$. Optimal bladder residual urine volume is less than $100 \mathrm{ml}$.

\section{Modulation of bladder and pelvic muscle function with pens (Fig. 1)}

As the bladder fills from the kidneys and ureters, the stretch receptors in the bladder dome wall send activation signals to the spine by way of the lower thoracic and upper lumbar nerve roots. Upon entry into the spinal cord, the information is processed locally and also directed to the brain stem. As the filling signals increase, the brainstem and spinal cord circuits increase in activity. Sub-cortical circuits inhibit the increasing spinal activity temporarily (inhibition reflex). The cortical brain identifies this increased activity and makes an additional decision to relax the autonomic system (detrusor) and tighten the 
pelvic floor and urethral sphincter muscles.

Active voiding requires sympathetic tone reduction to the urethral sphincter and a voluntary relaxation of the pelvic floor muscles. This produces unobstructed funneling as the bladder muscle (detrusor) contracts. This action can be considered similar to the action of agonist and antagonist muscles, which fire according to specific basic EMG patterns known as bi-phasic and tri-phasic EMG patterns. Voiding dysfunction may occur when the pattern of firing of the detrusor and sphincter go out of synchronization (i.e. co-contract or co-relax). This is referred to as Detrusor Sphincter Dyssynergy or DSD ${ }^{3)}$. This dysfunction may result from the alteration of normal spinal loop central pattern generators because of neural sensitization or changes in descending control.

One of the potential mechanisms of PENS is the re-establishment of correct timing of the central pattern generators. This would occur because of repetitive afferent stimulation using correctly timed tri-phasic EMG patterns that replicate normal agonist/antagonist motor patterns. This motor re-learning is referred to as muscle re-education and is thought to play a major role in such treatments as therapeutic exercise, continuous passive motion and proprioceptive neuromuscular facilitation $(\mathrm{PNF})^{8}$. Based on the review of the DSD case study, the urination hesitancy time was reduced and stream average $\mathrm{ml} / \mathrm{sec}$ improved. Urination hesitancy time may be related to spinal loop "boot-up" of the correct algorithm for voiding. If this is the case, improved timing coordination between detrusor and sphincters/pelvic floor muscles complexes may be a result of the motor re-education with PENS.

The precise patho-physiology of "urge" incontinence is not fully understood. It is distinct from "stress" and "neurogenic" incontinence in that the patient notes a sense of urgency that is difficult to control despite relatively low bladder filling volumes. This may occur due to bladder detrusor sensory and nocciceptive afferent sensitization. A minimally functional descending control and local reduced afferent input (perhaps due to reduced activity levels associated with institutionalized patients), which would normally gate the nocciceptive stretch receptors of the bladder dome, may also be primary factors in urge incontinence. Activation of stretch receptors triggers the parasympathetic (S-2, -3, -4) muscarinic (M2) acetylcholine receptors in the bladder wall, causing the detrusor to contract while simultaneously increasing bladder pressure. This activation is normally overridden or inhibited by descending fibers from the Periaqueductal Grey (PAG) region of the midbrain. Additional inhibition is created by contraction of the pelvic floor and external urethral sphincter muscles through sacral afferents. These are also triggered by increased proprioceptive input from the abdomen as intra-abdominal pressure increases due to normal and rapid movement, such as coughing. The balance between sensory and motor components of the sympathetic and parasympathetic systems maintains appropriate hydrostatic pressures and muscle tone during bladder filling and rapid movement and inhibits urge sensation. However, in older institutionalized patients, reduced mobility and activity decreases sensory input and thus the normal modulation of urge sensation and detrusor tone. This can lead to a rapid reflexive event of incontinence. The cascade is proposed as follows: 1 . the sensitized stretch receptors signal filling; 2 . this triggers sensation of urge ; 3 . the detrusor contracts, due to wind-down of central descending control and lack of local gating, overriding the sphincters and pelvic floor muscles (which may be out of synch due to disuse atrophy and central pattern generator dyssynergy). Urine flows and incontinence results. 
The neurons sending stretch receptor information from the detrusor have a high number of Kappa receptors at the spinal cord level ${ }^{9,10}$. The primary Kappa receptor agonist is a neurotransmitter known as dynorphin, which is associated with gate control modulation at the spinal level and is under the descending control of the PAG. One of the effects of PENS stimulation is the activation of sensory A Beta fibers at a frequency of $50 \mathrm{~Hz}$ during the bursts of stimulation ${ }^{11}$. This stimulation is known to trigger Dynorphin and Enkephalin through inhibitory spinal neurons located in the dorsal horn of the spinal cord ${ }^{9}$. It is proposed that part of the effect of PENS on urge suppression is the modulation of the kappa agonist dynorphin through sensory stimulation at the spinal levels associated with detrusor stretch receptor inputs ${ }^{10,12-14}$. The sensation of urgency is produced by ascending sensory and nocciceptive afferent sympathetic activity that is normally inhibited spinally, and at the pons and PAG (described as the inhibitory reflex). PENS may also trigger descending control from the PAG due to the low frequency components of the afferent volleys or bursts of neural signals, which stimulate A-delta fibers. These bursts at rates of $1-15 \mathrm{~Hz}$ trigger descending serotonergic activation of the Dynorphin inter-neurons and modulation of painful afferent inputs ${ }^{13)}$ (such as urge). Elderly patients and those with underlying neurologic conditions that result in a loss of bladder inhibitory control may improve their function with the application of sensory-motor re-education with PENS into the spinal segments that are components of the voiding reflex $\operatorname{arc}^{15} 20$ '.

A third and interesting finding in the frail elderly with urge or mixed incontinence is the relationship of the lack of micturition sensation and the development of incontinence. Patients may not sense fullness or urge due to malfunction of primary afferent pathways as a result of prior neurologic disease, denervation associated with childbirth or trauma ${ }^{21}$. Although the patient cannot feel the bladder stretch, the stretch receptors of the detrusor finally trigger voiding without the patient's knowledge. The patients just find themselves wet. The mechanism is the same as classically described urge incontinence but without the urge sensation. It is proposed that both hypo- and hyperactive levels of sensitization lead to incontinence in frail elderly patients. Either, the patients feel the urge sensation strongly but are unable to modulate or inhibit bladder contractions or they do not feel the sensation and void reflexively. In both cases the primary malfunction is likely to be neurologic either lack of afferent input or lack of inhibition or both. Findings from clinical trials in Japan (personal communications T. Fujiwara, date) have demonstrated that PENS stimulation is not effective in reducing incontinence in patients who are lacking or do not recover micturition sensation during the course of treatment. This is consistent with findings in United States studies and is illustrated with the neurogenic bladder case study. In this case, there was no initial sensation of fullness or micturition. During the course of treatment with PENS, the sensation slowly returned as evidenced by the changes in electro stimulation thresholds and patient reporting. Co-incident with the return of micturition sensation the patient began to resume normal bladder control. The same return of sensation is often found using PENS to treat neurologic disorders such as MS or stroke. It is postulated that micturition sensation and proprioceptive neuromuscular control are key issues in the treatment of mixed and urge incontinence in elderly institutionalized patients. As patients age or take various steroid drugs, the calcium channels of motor and sensory nerves may block up due to the development of antibodies at $\mathrm{CA}+$ receptor sites. Electrically induced low frequency voltage transients may dislodge these antibodies and increase neural 
activity $^{22}$. It is postulated that PENS assists in the regaining of sensation and motor control partially through the application of appropriately patterned pulses at voltage levels capable of clearing $\mathrm{CA}+$ channels $^{22}$. Many older patients may also be receiving $\mathrm{CA}+$ channel blockers, which further contribute to the development of incontinence. The neurologic wind down due to lack of movement, behavior modification related to the use of protective garments or pads, and the effect of inappropriately administered anticholinergic medications or other urge inhibitors may be primary factors in the development of institutionalized urge and mixed incontinence ${ }^{3)}$.

Differential diagnosis using urodynamic testing is important in deciding the plan of care as evaluation purely on symptoms may provide a very different diagnosis ${ }^{31}$. For example, the urodynamic evaluation of detrusor hyperactivity with impaired contractility would indicate Ditropan was not appropriate. vs. detrusor hyperactivity with normal contractility where an urge suppressant may be appropriate (both provide similar symptoms on history taking without urodynamics).

\section{Pelvic floor muscle strengthening with mfac (Fig. 2)}

Stress incontinence may be associated with intrinsic sphincter dysfunction (ISD). The primary mechanism in non-denervated patients is proposed as neural dyssynergy coupled with muscle disuse atrophy ${ }^{20 !}$. Sensory inputs associated with increased intra-abdominal pressure normally trigger reflex urethral closing pressure through the sphincter and pelvic floor muscles. If this timing loop is slow or out of synch compared to the rate of rise of abdominal pressure, urinary leakage occurs. This may also be coupled with muscle disuse atrophy. Disuse Atrophy of the muscle units generally starts with fast twitch motor units ${ }^{6,23}$ responsible for the fast closing of the sphincters and approximation of the pelvic floor ${ }^{21}$. Fast twitch muscle atrophy manifests itself with aging and is more pronounced in the sedentary lifestyles associated with the institutionalized elderly. A muscle can atrophy with or without its peripheral nerve supply intact. By far the most commonly seen atrophy in physical medicine is due to an innervated muscle's lack of contractile activity or disuse.

An alternative or additional method of treating disuse atrophy is the use of electrical stimulation. Electrical stimulation can be used to activate muscles either in the absence of, or when superimposed upon concurrent voluntary efforts to activate the muscle. The pelvic floor muscle of primary interest is the pubococcygeus portion of the levator ani ${ }^{23)}$. The pubococcygeus muscle consists of a mixture of Type I slow-twitch (70\%) and Type II fast-twitch $(30 \%)$ muscle fibers innervated by the pudendal nerve $(\mathrm{S} 2-\mathrm{S} 4)^{24}$. Histological studies on women with urinary incontinence have shown a $15 \%$ to $30 \%$ decrease in Type II fast-twitch fibers compared to symptomatic women ${ }^{2,25}$. This loss of Type II fibers decreases the muscle's ability to produce fast powerful contractions designed to increase urethral pressure, thus preventing involuntary loss of urine during coughing, sneezing, laughing, physical exertion, or unstable detrusor contraction ${ }^{26}$.

One way to improve urethral closure pressure and urinary control is by preferentially strengthening the type II fast-twitch fibers of the pubococcygeus muscle. This is best accomplished with electrical stimulation and not voluntary contraction, since electrical stimulation preferentially activates type II fast-twitch fibers ${ }^{8-11}$. In addition, the stimulation has been shown to improve the muscle thickness and capillary bed of the pelvic floor muscle both postulated to be important components associated with disuse atrophy ${ }^{26,27)}$. 
Medium Frequency Alternating Current (MFAC) therapy can directly stimulate contractions of the pelvic floor resulting in muscle strengthening. Our study supports the use of this therapy for treatment of urge and mixed incontinence. MFAC is appropriate for the treatment of incontinence in the elderly patient as it provides a comfortable non-invasive method of treating disuse atrophy of the pelvic floor musculature, which is common in all patients regardless of the cause of incontinence. An appropriate evaluation method would be to use EMG spectral and amplitude analysis to determine disuse atrophy (as the frequency spectrum demonstrates whether slow or fast twitch fibers are affected), and subsequent strengthening during a course of treatments. MFAC treatment can provide the following additional advantages :

1. Identifying and contracting the pelvic floor muscles because they are not visible and routinely used. Women must be taught how to correctly isolate and contract pelvic floor muscles. Studies have shown that approximately $40 \%$ of women are unable to effectively recruit or isolate the pelvic floor muscles following simple verbal instructions ${ }^{25,27)}$. This percentage increases dramatically with age and neural desensitization. The feeling of the electrical stimulation induced contraction of the pelvic floor muscle is a useful reminder to the patient of the sensation that should be perceived during voluntary contraction of the muscle.

2. In cases of severe muscle weakness (zero to fair grades), where the patient cannot actively contract against gravity, electrical stimulation can assist in re-establishing basic strength and motor control of the pelvic floor and associated muscle groups by improving postural tone and pelvic floor power.

3. The results of electrical stimulation are less dependent on patient cooperation and motivation than with voluntary exercise ${ }^{25,27)}$. This is of particular importance in re-educating function in elderly patients who may suffer from some degree of dementia.

4. Pelvic floor exercises and re-education with or without electrical stimulation is one of the most cost effective methods of achieving pelvic floor rehabilitation and a viable alternative to surgical intervention ${ }^{4)}$.

\section{Electrical stimulation re-education via pens}

The objective is to re-educate pelvic floor and sphincter muscle control, alter sensitization and improve nerve and muscle atrophy in the elderly with urge, stress and mixed incontinence as well as those with neurogenic impairments. We have shown in this study that long term care patients with incontinence showed significant success in reducing the number of episodes of urge or mixed incontinence. Previous work by us has shown that PENS is also effective in treating neurogenic incontinence, but that stress incontinence may require further treatment with pelvic floor exercise, MFAC and biofeedback ${ }^{25}$.

\section{Why not just use MFAC}

In our study, patients who underwent both PENS and MFAC treatments and the PENS only treatment showed significant improvements in UI. Both groups showed similar results within the standard deviation of the data. The study designs were based on the postulate that PENS was required initially to re-educate motor sensory control loops, modulate sensitization and improve micturition sensation. This was based on the observation that the majority of elderly UI patients lack the sensation that warns them when the bladder is full 
or cannot fully sense micturition. The PENS technique helps to re-educate sensory motor loops, modulate the sensory nerves to this area and wake up the motor nerves. We believe that if we had used only MFAC, our results would not be as good, as most institutionalized elderly have significant neural and muscle atrophy, which impedes the ability to effectively elicit muscle contraction with MFAC. (Our prior clinical experience also demonstrated that starting with an aggressive motor stimulation strategy increases urinary and bowel incontinence, which was unacceptable to most patients. A less aggressive treatment plan with a gradual return to function is indicated in this patient population and results in reduced incontinence.) In exit interviews, patients stated that after only a few treatments with PENS, their sensation of having to urinate was heightened. Based upon our prior experience with MFAC side effects early in the course of treatment, and the fact that we wanted all patients to get better, we did not employ an MFAC only treatment.

Our results could be supported further and added upon by a different study design. Future studies could be designed with three groups : one that receives only PENS, one that receives only MFAC, and a control group that receives a sham treatment (hook up the electrodes to the unit, but do not turn it on). This could either lend support to or refute our claims that PENS is the essential ingredient in the treatment of patients with urinary incontinence. We suggest that additional studies systematically use urodynamic testing and the reporting forms developed by the end of the studies to further link specific diagnosis to clinical outcomes.

\section{Conclusions}

PENS, a gentle form of EMG Patterned Electrical Neuromuscular Stimulation, applied to the pelvic musculature is a comfortable, non-invasive treatment for urge or mixed incontinence in elderly nursing home residents. Overall, the results of this study demonstrate a reduction of incidence of urge and mixed urinary incontinence by an average of $72.5 \%$ following a course of six weeks of stimulation with PENS. A combination approach using PENS and MFAC (PENS for the first three weeks and a combination of PENS and MFAC for the last three weeks for a total six-week course of treatment) was also tested and was found to reduce urinary incontinence by $66 \%$ in both urge and mixed incontinence patients. This method may be more effective in treating cases with a more distinct stress incontinence component, as the stronger motor stimulation produced by medium frequency alternating current (MFAC) causes a more intense pelvic muscle contraction with a corresponding increase in muscle strength.

From a clinical perspective, PENS, MFAC and PME (Pelvic Muscle Exercise) are important in reducing muscle and nerve disuse atrophy, neural sensitization and for re-educating neuro-muscular control loops associated with the maintenance of urinary continence. In medically complex institutionalized elderly, incontinence is a multifactorial problem and must be managed as such. PENS and MFAC provide additional clinically effective modalities for the therapeutic improvement of incontinence in institutionalized elderly patients.

Effective urinary incontinence programs in long-term care facilities should incorporate rehabilitation treatments using PENS, MFAC and PME in combination with the putting in place of a multi-disciplinary team who monitor, manage and provide appropriate care for their patients with incontinence. The enhancement of the patient's quality of life and their 
increased participation in activities of daily living, the reduced cost of skin breakdown, laundry, adult incontinence products and additional nursing care, provide an attractive, cost effective treatment option for the management of incontinence in the elderly.

\section{References}

1) Hu TW : The economic impact of urinary incontinence. Clin Geriatric Med 2: 673-687 (1986)

2) Gilpin SA, Gosling JA, Smith ARB and Warrell DW: The pathogenesis of genitourinary prolapse and stress incontinence of urine. A histological and histochemical study. Br J Obstet Gynaecol 96 : 15-23 (1989)

3) Geriatric urodynamics. In : Atlas of Urodynamics. Blaivas $\mathbf{J}$ and Chancellor $\mathbf{M}$ (Eds), Williams and Wilkins, Philadelphia, USA, pp 271-282 (1996)

4) US Department of Health and Human Services: Managing Acute and Chronic Urinary Incontinence, Quick reference guide for clinicians, in AHCPR publication number 96-0686 (1996)

5) Dumoulin C, Seaborne DE, Quirion-DeGirardi $C$ and Sullivan SJ: Pelvic-floor rehabilitation, Part 1: Comparison of two surface electrode placements during stimulation of the pelvic-floor musculature in women who are continent using bipolar interferential currents. Phys Ther 75 : 1067-1074 (1995)

6) Sinacore D, Delitto A, King D and Rose S: Type II fiber activation with electrical stimulation. A Preliminary report. Phys Ther $70: 416-422$ (1990)

7) Innervation of the Kidneys, Ureters and Urinary Bladder. In: The Ciba Collection of Medical Illustrations, Vol. 1- Nervous System, Netter FH, Brass A and Dingle RV (Eds), Ciba-Geigy Corporation, West Caldwell, N.J., pp 86-87 (1983)

8) Trimble $M$ and Enoka $R$ : Mechanism underlying the training effects associated with neuromuscular Electrical stimulation. Phys Ther $71: 273-282$ (1991)

9) Sun SL and Han JS: High and low frequency electro acupuncture analgesia are mediated by different types of opioid receptors at spinal level: a cross tolerance study. Sheng Li Xue Bao 41 : 416-420 (1989)

10) Xin Su, Sengupta JN and Gebhart GF : Effects of opioids on mechanosensitive pelvic nerve afferents of the rat. Proceedings of the 8th World Congress on Pain, Progress in Pain Research and Management, Vol. 8, Jenson TS, Turner JA and Wiesenfeld-Halin Z (Eds), IASP Press, Seattle pp 1566-1580 (1997)

11) Han JS, Chen XH, Sun SL, Xu XJ, Yuan Y, Yan SC, Hao JX and Terenius L: Effect of low and high frequency TENS on Met-enkephalin-Arg-Phe and dynorphin A immunoreactivity in human lumbar CSF. Pain 47 : 295-298 (1991)

12) Elie D, Al-Chaer, Westlund Katin $\mathbf{N}$ and Willis WD: The Dorsal Column: A possible role in viseral hyperalgesia. In : Proceedings of the $8^{\text {th }}$ World Congress on Pain, Progress in Pain Research and Management, Vol. 8, Jenson TS, Turner JA and Wiesenfeld-Halin Z (Eds), IASP Press, Seattle pp 120-124 (1997)

13) Hisamitsu $T$ and De Groat WC: The inhibitory effect of opioid peptides and morphine applied intrathecally and intracerebroventricularly on the micturition reflex in the cat. Brain Res 298 : 51-65 (1984)

14) De Groat WC and Kawatani M: Enkephalinergic inhibition in parasympathic ganglia of the urinary bladder of the cat. J. Physiol (Lond) $413: 13-29$ (1989)

15) Chen XH and Han JS : All three types of opioid receptors in the spinal cord are important for $2 / 15 \mathrm{~Hz}$ electro acupuncture analgesia. Eur J Pharmacol 211 : 203-210 (1992)

16) Tahseen Hasan, Robson Wendy, Pridie Angus $\mathrm{K}$ and Neal David E : Transcutaneous electrical stimulation and temporary S3 neuromodulation in idiopathic detrusor instability. J Urol 155 : 2005-2011 (1996)

17) Nakamura M, Sakurai T, Tsujimoto $Y$ and Tada $Y$ : Transcutaneous electrical stimulation for the control of frequency and urge incontinence. Acta Urol Jpn 29 : 1053-1059 (1983) (in Japanese)

18) McGuire EJ, Zhang SC, Horwinski ER and Lytton B : Treatment of motor and sensory detrusor instability by electrical stimulation. $J$ Urol 129 : 78-79 (1983)

19) Vodusek DB, Light JK and Libby JM : Detrusor inhibition induced by stimulation of pudendal nerve afferents. Neurourol Urodyn 5 : 381-389 (1986)

20) Mauroy B, Devillerts P, Demetriou D, Ametepe B and Biserte J: Treatment of bladder instability with interferential current. Report of 20 cases: preliminary results. Prog Urol 2 : 664-670 (1992)

21) Bernstein IT : The pelvic floor muscles : muscle thickness in healthy and urinary-incontinent women measured by perinea ultrasonography with reference to the effect of pelvic floor training. Estrogen receptor studies. Neurourol Urodyn 16 : 237-275 (1997)

22) Raymond C, Walker D, Bichet D, Iborra C, Martin-Moutot N, Seagar M and De Waard M: Antibodies against the beta subunit of voltage-dependent calcium channels in Lambert-Eaton myasthenic syndrome. Neuroscience 90 : 269-277 (1999)

23) Knaflitz M, Merletti $R$ and DeLuca $C$ : Influence of motor unit recruitment order in voluntary and electrically 
elicited contractions. J Appl Physiol 68 : 1657-1667 (1990)

24) Gosling J, Dixon J, Critchly $\mathrm{H}$ and Thompson S : A comparative study of the human external sphincter and periurethral levator ani muscle. Br J Urol 53 : 35-41 (1981)

25) Sand PK, Richardson DA, Staskin DR, Swift SE, Appel RA, Whitmore KE and Ostergard DR : Pelvic floor electrical stimulation in the treatment of genuine stress incontinence: A multicenter, placebo-controlled trial. Am J Obstet Gynecol 173 : 72-79 (1995)

26) Miller JM, Kasper C and Sampselle C: Review of muscle physiology, with application to pelvic muscle exercise. Urol Nurs 14 : 92-97 (1994)

27) Blowman C, Pickles C, Emery S, Creates V, Towell L, Blackburn N, Doyle N and Walkden B : Prospective double blind controlled trial of intensive physiotherapy with and without stimulation of the pelvic floor in treatment of genuine stress incontinence. Physiotherapy $77: 661-664$ (1991)

[Received November 25, 2001 : Accepted September 25, 2004] 